\title{
Observational Medical Outcomes Partnership
}

National Cancer Institute

\section{Source}

National Cancer Institute. Observational Medical Outcomes Partnership. NCI Thesaurus.

Code C127113.

A public-private partnership established to inform the appropriate use of observational healthcare databases for studying the effects of medical products. Its goals were: 1) conduct methodological research to empirically evaluate the performance of various analytical methods on their ability to identify true associations and avoid false findings, 2) develop tools and capabilities for transforming, characterizing, and analyzing disparate data sources across the health care delivery spectrum, and 3) establish a shared resource so that the broader research community can collaboratively advance the science. 